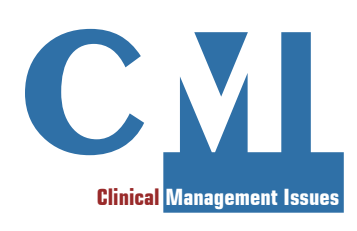

\title{
Histoplasmosis in Italy
}

L'istoplasmosi in Italia

CMI 2018; 12(1): 49-51

https://doi.org/10.7175/cmi.v12i1.1369

In loving memory of Professor Aldo Mazzoni, MD, 1927-2007

\section{HISTOPLASMOSIS}

Histoplasmosis is a fungal infection caused by Histoplasma capsulatum var. capsulatum and Histoplasma capsulatum var. duboisii (in Africa) [1].

These infective agents are present mainly in the soil, particularly if contaminated with bird or bat droppings. The infection, acquired via inhalation, is generally asymptomatic, but in a small percentage of cases (1\%) it may result in influenza-like symptoms, often self-limiting. It affects mainly immunocompromised patients and worsens in case of high exposure level. It may involve all organs and systems, and may mimic many other infectious and non-infectious disorders, which are more frequent just in these immunocompromised hosts. In the most severe cases, disseminated histoplasmosis may be life-threatening [1].

Among systemic and cutaneous mycoses diffused worldwide, histoplasmosis is still a present threat for people living in endemic countries, travelers, immigrants, and in some cases also in non-endemic countries, especially when immunocompromised patients are of concern.

The most widely used diagnostic tests are Histoplasma antigen detection in urine and/ or serum, but also culture, antibody tests, and microscopy may be performed, even if each of them present important drawbacks [1].
Histoplasmosis belongs to a particular group of pathogenic fungi, which are called "dimorphic", since they present as yeasts at body temperature $\left(37^{\circ} \mathrm{C}\right)$ and as fungal hyphae at room temperature $\left(25^{\circ} \mathrm{C}\right)$. This distinction is also very useful for the standard diagnostic techniques, since it is sufficient to change the temperature of the culture vial to allow the observation of this relevant morphologic change.

In cases of mild to moderate pulmonary histoplasmosis, the infection will resolve spontaneously, without treatments. For severe infections, an effective antifungal therapy (amphotericin B or itraconazole) is available, but a prompt diagnosis plays also a significant role in rapidly achieving a complete cure [1,2].

\section{THE DIAGNOSIS OF HISTOPLASMOSIS IN ITALY}

Since 1950s, histoplasmosis was retrieved in the Po river valley. Most cases concerned imported histoplasmosis, but also native histoplasmosis was found, as confirmed by studies on soil [3] and animals [4]. From 1955 to 1960,7 cases of native histoplasmosis were reported in the Emilia Romagna, Piedmont, and Venetia regions [5]. Afterwards, no cases of native histoplasmosis were published until 1989.
Corresponding author Prof. Roberto Manfredi, MD Infectious Diseases, Department of Medical and Surgical Sciences, "Alma Mater Studiorum" University of Bologna, S. Orsola Hospital Via Massarenti, 11

I-40138 Bologna, Italy Telephone: +39051214.3355 Telefax: +39051343500

E-mail: roberto.manfredi@unibo.it

Received: 26 March 2018

Accepted: 16 July 2018

Published: 20 July 2018 
During mid-1980s, the number of episodes of histoplasmosis among immunocompromised patients (especially those with HIV infection and AIDS) [6] grew and the number of cases of histoplasmosis occurring among travelers increased. At that time, the diagnosis of histoplasmosis was still based on the standard techniques, and missed or delayed diagnosis were common, when a skilled expertise in this field was lacking.

The diagnostic problems dealing with the frequent cutaneous localization of histoplasmosis [7] became even more difficult in immunocompromised patients. Particularly hard was the differential diagnosis with other opportunisms and underlying disorders $[8,9]$. Cutaneous forms may be especially challenging, and often require histopathologic studies to achieve a diagnosis, which leads to an appropriate clinical treatment.

A paper published in 2005 [5] calculated 55 cases of histoplasmosis reported in Italy in around 50 years. 20 of these patients were coinfected by HIV. Native histoplasmosis was detected in 13 cases, that were concentrated in the Northern regions and in the most recent years.

Lately, more advanced molecular biology techniques to obtain diagnosis of histoplasmosis have been developed: mycoarrays [10].
It comes of a serological assay built up on a protein microarray platform specifically developed to detect antibodies against histoplasmin (for the diagnosis of histoplasmosis) and other antigens of Coccidioides immitis (for the diagnosis of coccidioidomycosis).

In my opinion, especially when imported histoplasmosis does not appear to have a lifethreatening course, it continues to be borne by very relevant epidemiological, clinical, imaging, and histopathologic clues. Tissue samples available after biopsy may be needed for an histopathological examination, and especially a relevant history and a diagnostic suspicion are non-negligible clues, as just in a recently described Italian traveler who acquired imported histoplasmosis after his trip in the Mato Grosso region, Brazil [11].

Finally, I believe that the traditional mycological techniques (including microscopy and culture at the two key different temperatures where Histoplasma spp. show their yeast and hyphal forms, respectively), especially in conjunction with a timely diagnostic suspicion, may allow to expect that an endemic, imported (but sometime autochthonous) mycosis like histoplasmosis can be easily and promptly recognized also in Institutions where extremely advanced laboratory techniques are not still available.

The role of Professor Aldo Mazzoni

The University of Bologna represents since over 60 years a national reference center for dimorphic mycoses, especially for both imported and native histoplasmosis. H. capsulatum, in fact, was retrieved in the Po river valley since 1950s by Professor Aldo Mazzoni and other researchers. He carried out systematic studies [3,4,6,12-14] in humans, animals, and in soil specimens, recognized also by a report published in Science in the year 1965 [3], among others. His personal engagement covered several vocations: he was clinician, microbiologist, mycologist, and finally bioethical expert [3,4,6,13-15]. His expertise, in particular in the field of histoplasmosis, was widely recognized, to the point that he frequently acted as "second opinion" for cases coming from the entire country.

During mid-1980s, the University of Bologna carefully collected and discussed all cases of histoplasmosis capsulatum and duboisii reported by the scientific literature since the year 1980, distinguishing them from their presumably native or imported origin [16]. At that time, when a skilled expertise in this field was lacking, this systematic work has been acknowledged also by many institutional, epidemiological organisms, in Italy and abroad. Prof. Mazzoni gave also his valuable contribution in solving the diagnostic problems dealing with the frequent cutaneous localization of histoplasmosis [7] in the immunocompromised patients, which required a differential diagnosis with other opportunisms and underlying disorders [8,9].

\section{Funding}

This article has been published without the support of sponsors.

Conflicts of Interest

The author declares he worked in Professor Mazzoni's team described in the article. 


\section{REFERENCES}

1. Centers for Disease Control and Prevention. Information for Healthcare Professionals about Histoplasmosis. Available at https://www.cdc.gov/fungal/diseases/histoplasmosis/healthprofessionals.html (last accessed April 2018)

2. Wheat LJ, Freifeld AG, Kleiman MB, et al; Infectious Diseases Society of America. Clinical practice guidelines for the management of patients with histoplasmosis: 2007 update by the Infectious Diseases Society of America. Clin Infect Dis 2007; 45: 807-25; https://doi. org/10.1086/521259

3. Sotgiu G, Mazzoni A, Mantovani A, et al. Histoplasma capsulatum: occurrence in soil from the Emilia Romagna region of Italy. Science 1965; 147: 624; https://doi.org/10.1126/ science.147.3658.624

4. Mantovani A, Mazzoni A, Ajello L. Histoplasmosis in Italy. Isolation of Histoplasma capsulatum from dogs in the province of Bologna. Sabouraudia 1968; 6: 163-4; https://doi. org/10.1080/00362176885190291

5. Farina C, Rizzi M, Ricci L, et al. Imported and autochthonous histoplasmosis in Italy: new cases and old problems. Rev Iberoam Micol 2005; 22: 169-71

6. Mazzoni A, Pileri S, Manfredi R. Le micosi sistemiche. In: Ajello L, Farina C, Mazzoni A, et al. (eds.). Fondamenti di Micologia Clinica. Milan: AMCLI, 1993, pp. 357-69

7. Chang P, Rodas C. Skin lesions in histoplasmosis. Clin Dermatol 2012; 30; 592-8; https://doi. org/10.1016/j.clindermatol.2012.01.004

8. Manfredi R, Mazzoni A, Pileri S, et al. Simultaneous occurrence of visceral leishmaniasis and disseminated histoplasmosis in an Italian patient with HIV infection. Infection 1994; 22: 2245; https://doi.org/10.1007/BF01716715

9. Calza L, Manfredi R, Donzelli C, et al. Disseminated histoplasmosis with atypical cutaneous lesions in an Italian HIV-infected patient: another autochtonous case. HIV Medicine 2003; 4: 145-8; https://doi.org/10.1046/j.1468-1293.2003.00144.x

10. Ardizzoni A, Baschieri MC, Manca L, et al. The protein "mycoarray": a novel serological assay for the laboratory diagnosis of primitive endemic mycoses. New Microbiol 2011; 34: 307-16

11. Ardizzoni A, Baschieri MC, Manca L, et al. The mycoarray as an aid for the diagnosis of an imported case of histoplasmosis in an Italian traveler returning from Brazil.JTravel Med 2013; 20: 336-9; https://doi.org/10.1111/jtm.12060

12. Sotgiu G, Corbelli G. Rare mycosis: study of the first two cases of histoplasmosis in Italy and a case of coccidioidomycosis. Bull Sci Med (Bologna) 1955; 127: 85-92

13. Corbelli G, Mazzoni A, Allegri L. The additional cases oh histoplasmosis as observed in Bologna Medical Clinic. Minerva Med 1957; 148: 3823-6

14. Mantovani A, Mazzoni A. Histoplasmosis in Italy. Bull Sci Med (Bologna) 1971; 143: 57-69

15. Manfredi R, Mazzoni A. Le micosi sistemiche. In: Ajello L, Farina C, Mazzoni A, et al. (eds.). Fondamenti di Micologia Clinica. Milan: AMCLI, 1993, pp. 373-92

16. Manfredi R, Mazzoni A, Nanetti A, et al. Histoplasmosis capsulati and duboisii in Europe: the impact of the HIV pandemic, travel and immigration. Eur J Epidemiol 1994; 10: 675-81; https://doi.org/10.1007/BF01719280 